\title{
Survey of Mycoplasma pneumoniae in Iranian children with acute lower respiratory tract infections
}

\begin{abstract}
Objectives: Mycoplasma pneumoniae is an atypical pathogen, which is one of the major causes of lower respiratory tract infections (LRTIs) worldwide. This study was performed to determine the role of M. pneumoniae in acute LRTIs in children, who were referred to main pediatric hospitals in Shiraz, Iran, with the diagnosis of LRTI. Polymerase chain reaction method on a throat-swab specimen was utilized to detect M. pneumoniae. Results: One hundred patients with acute LRTIs were investigated in this study. There were 10 positive PCR for M. pneumoniae (10\%), including 6 of 62 hospitalized patients and 4 of 38 outpatients. All patients with LRTIs due to M. pneumoniae had cough. Fever, flu like symptoms, dyspnea, pulmonary rales, wheezing, and conjunctivitis were other common signs and symptoms. Conclusions: The percentage of cases with M. pneumoniae infection in our population is similar to the reported in other parts of Asia. Precise and early detection of pathogen and appropriate antibiotic therapy are the key points in management of patients with LRTIs.
\end{abstract}

Keywords: atypical bacteria; Mycoplasma pneumoniae; PCR; respiratory infection;

[Braz J Infect Dis 2011;15(2):97-101 ] $\odot$ Elsevier Editora Ltda.

\section{INTRODUCTION}

Lower respiratory tract infections (LRTIs) continue to be a common cause of morbidity and mortality, especially in the pediatric age group, ${ }^{1,2}$ contributing to about 1.9 million children's deaths yearly. ${ }^{3}$ Determination of the putative pathogens is one of the main difficulties in LRTIs, as the diagnostic tests of respiratory samples are not sensitive enough to identify the causative microorganisms in most patients. ${ }^{4,5}$ It is estimated that antigen screening, culture and serological methods can be helpful in only one third of the cases. ${ }^{6}$ Therefore therapy is usually empiric in most cases. ${ }^{7,8}$

Atypical pathogens, particularly $\mathrm{Myco}$ plasma pneumoniae, are major causes of acute LRTIs. Although M. pneumoniae usually causes pneumonia and bronchitis, other clinical manifestations such as rhinitis, pharyngitis, sinusitis and otitis media can also be seen. ${ }^{9,10}$ There are some reports indicating significant role of M. pneumoniae as cause of LRTIs in children of all ages, ${ }^{11,12}$ which underscores the importance of specific pathogen diagnosis, as the use of proper antibiotics, such as macrolides, can significantly reduce duration of the illnesses. ${ }^{13,14}$
Unfortunately, as result of the difficulty to $\operatorname{detect} M$. pneumoniae due to insensitive culture and time-consuming and impractical in clinical practice, specific etiologic diagnoses have remained unknown in the majority of cases. ${ }^{15}$ Several methods were utilized for detection of this pathogen, including cold agglutination test, ${ }^{16}$ serological methods (Mycoplasma IgM antibody, complement fixation, ELISA $)^{17,18}$ and microparticle agglutination test. ${ }^{19}$ However, there were a number of limitations with these methods, especially regarding sensitivity and specificity. Therefore polymerase chain reaction (PCR) is suggested as of the most practical typing method for rapid and sensitive detection of M. pneumoniae in throat swabs. ${ }^{20,21}$ The PCR method could provide rapid diagnosis of $M$. pneumoniae infection, especially in younger children, and is a valuable method considering epidemiological, clinical, economic aspects in addition to turnaround time of results. ${ }^{22-24}$ The sensitivity and specificity of the PCR method has been estimated about $70 \%$ and $80 \%$, respectively. ${ }^{24}$

This study was performed to determine the role of M. pneumoniae in acute LRTIs in children, with use of PCR analysis.
Authors

Nahal Hadi ${ }^{1}$

Sara Kashef ${ }^{2}$

Mohammad Moazzen ${ }^{3}$

Michel Shamoon Pour ${ }^{4}$

Nima Rezaei ${ }^{5}$

${ }^{1}$ Department of Bacteriology and Virology, Medical School Shiraz University of Medical

Sciences, Shiraz, Iran

${ }^{2}$ Allergy Research Center,

Department of Pediatrics,

Division of Immunology and

Allergy, Namazi Hospital, Shiraz

University of Medical Sciences,

Shiraz, Iran

${ }^{3}$ Department of Pediatrics,

Namazi Hospital, Shiraz

University of Medical Sciences,

Shiraz, Iran

${ }^{4}$ Department of Bacteriology

${ }^{4}$ Department of Bacteriology
and Virology, Medical School,

Shiraz University of Medical

Sciences, Shiraz, Iran

${ }^{5}$ Growth and Development

Research Center, Tehran

University of Medical Sciences,

Tehran, Iran Pediatrics Center

of Excellence, Children's

Medical Center, Tehran

University of Medical Sciences,

Tehran, Iran

Submitted on: $3 / 8 / 2010$ Approved on: 6/16/2010

Correspondence to:

Nahal Hadi

Academic Unit of Infection \&

Immunity,

School of Medicine \&

Biomedical Sciences, F Floor,

Medical School, University

of Sheffield, Beech Hill Road,

Sheffield, S10 2RX, UK

Phone: +44 7556020485

hadina@sums.ac.ir

We declare no conflict of interest. 


\section{PATIENTS AND METHODS}

\section{Setting}

This prospective study was performed in 2006 on pediatric patients, who were referred to Namazee and Dastgheib Hospitals (Shiraz, Iran) because of acute lower respiratory tract infections. This study was approved by Ethics Committee of the Hospital, Shiraz University of Medical Sciences. Written informed consent was taken from the parents of enrolled patients.

\section{Patients enrollment}

One hundred children (64 boys and 36 girls) were included in this study. Demographic data and patients' history were documented in the standardized questionnaire, designed for this study. All symptoms and detected signs in the physical examination, including cough, flu-like symptoms, dyspnea, pulmonary rales, wheezing, and conjunctivitis were also recorded. Initial laboratory investigations, including complete cell blood count and chest X-ray, were done before throatswab sampling. The patients with the following criteria were enrolled in this study: respiratory symptoms associated with abnormal chest X-ray suggestive of pneumonia, respiratory distress, and presence of rales in lung examination. Fever was defined as body temperature higher than $37.8^{\circ} \mathrm{C}$.

\section{Exclusion criteria}

Patients with chronic respiratory problems, such as asthma and cystic fibrosis, and other chronic diseases, such as malignant disease, tuberculosis and autoimmune diseases, were excluded from this study. Those who had history of blood transfusions or immunosuppressive therapy were also excluded. Enrolled patients were not receiving antibiotics during the 72 hours before sampling.

\section{PCR method}

The polymerase chain reaction (PCR) method on a throat-swab specimen was utilized to detect $M$. pneumoniae. Although all the patients were prescribed a 10 day course of macrolides (erythromycin or azithromycin at the recommended dose), throat-swab specimens were taken at the first day prior to antibiotic therapy. The samples were transported in PPLO broth to the microbiology laboratory and were incubated at $37^{\circ} \mathrm{C}$ for a few hours after removing the swabs. The cultures were centrifuged at 1,000 g for 5 minutes. The supernatant then was collected in a sterile $1 \mathrm{~mL}$ Eppendorf tube and centrifuged at 20,000 g for 20 minutes. After decanting the supernatant the pellet was suspended in $20 \mu \mathrm{L}$ of sterile Mili-Q water and boiled for 10 minutes.
The latter was then used as DNA template in PCR reactions which were performed by the M. pneumoniae PCR kit (Genekam Biotechnology AG, Germany), based on the protocol. The negative and positive controls provided in the kit were used in each assay. DNA fragments were visualized on $1 \%$ agarose gel by electrophoresis.

\section{Statistical analysis}

Epi Info 6 program (version 6.2, World Health Organization, Geneva, Switzerland) was used for statistical analyses. Association between categorical variables were assessed by Chi-square or Fisher's exact test. P-value of less than 0.05 was considered statistically significant.

\section{RESULTS}

\section{Characteristics of patients}

One hundred patients with acute LRTIs (64 male and 36 female), including 62 hospitalized and 38 outpatients, were investigated in this study. Patients' ages ranged from 6 months to 17 years.

\section{Positive PCR patients}

Among 100 investigated patients, PCR for M. pneumoniae turned out positive in $10(10 \%)$ patients, being 5 boys $(7,8 \%)$ and 5 girls $(13,8 \%)$ [p $=0.48]$. M. pneumoniae positivity rates among hospitalized patients $(9.5 \%)$ and outpatients $(10.5 \%)$ were similar $(\mathrm{p}=0.57)$.

\section{Seasonal variations}

Half of the identified $M$. pneumoniae cases were referred during the autumn season, while only 3 cases occurred during the winter and 2 cases in the spring. No M. pneumoniae was detected during the summer season.

\section{Clinical manifestations}

All patients with acute LRTI due to $M$. pneumoniae complained of cough lasting for a mean of 9.5 days, compared to a mean duration of 7 days among PCR negative patients $(\mathrm{p}=0.35)$. Although 8 out of 10 patients with positive PCR had fever at the time of admission, and all but one had low-grade fever. Half of the cases presented with productive cough, while the remaining half had dry cough, irrespective of being positive and negative PCR for $M$. pneumoniae. Flu-like symptoms were present in $40 \%$ of the patients with $M$. pneumoniae, which was lower than the $63.3 \%$ seen in the negative patients. However, this difference was not significant $(\mathrm{p}=0.18)$. In contrast, dyspnea was more common among PCR-positive patients for M. pneumoniae (50\% vs. $36.7 \%$, $\mathrm{p}=0.49$ ). Other characteristics of the patients are presented in the Table 1, which shows no significant difference on clinical manifestations between these two groups. 
Table 1. Characteristics of patients with acute lower respiratory tract infections

\begin{tabular}{|lccc}
\hline Characteristics & $\begin{array}{c}\text { PCR-Positive for M. pneumoniae } \\
(\mathbf{n = 1 0 )}\end{array}$ & $\begin{array}{c}\text { PCR-Negative for M. pneumoniae } \\
(\mathbf{n}=\mathbf{9 0})\end{array}$ & $\begin{array}{c}\text { p-value } \\
*\end{array}$ \\
\hline Gender (male/female) & $5 / 5$ & $59 / 31$ & 0.49 \\
\hline Cough & $10(100 \%)$ & $90(100 \%)$ & - \\
\hline Fever & $8(80 \%)$ & $57(63.3 \%)$ & 0.49 \\
\hline Flu like symptoms & $4(40 \%)$ & $57(63.3 \%)$ & 0.18 \\
\hline Dyspnea & $5(50 \%)$ & $33(36.7 \%)$ & 0.49 \\
\hline Pulmonary rales & $3(30 \%)$ & $34(37.8 \%)$ & 0.74 \\
\hline Wheezing & $1(10 \%)$ & $23(25.6 \%)$ & 0.68 \\
\hline Conjunctivitis & $2(20 \%)$ & $11(12.2 \%)$ & 0.34 \\
\hline Hospitalized cases & $6(60 \%)$ & $56(62.2 \%)$ & 0.57 \\
\hline Outpatient cases & $4(40 \%)$ & $34(37.8 \%)$ & 0.57 \\
\hline
\end{tabular}

*Not significant in all these comparisons.

\section{Para-clinical findings}

Complete blood cell count was similar in the two groups of positive and negative PCR for M. pneumoniae. Patients with positive PCR for $M$. pneumoniae had a mean white blood cells (WBC) count of $10,200 / \mathrm{m}^{3}$ with $65 \%$ polymorphonuclear cells (PMN), whilst mean WBC in the PCR-negative cases was $10,000 / \mathrm{m}^{3}$ with $54 \%$ PMN. Expert radiologist reports of chest X-ray were available for 70 patients; among PCR-positive patients for M. pneumoniae there were 2 cases of lobar infiltrations and 3 of interstitial infiltrations. Positive radiological findings among PCR-positive patients for M. pneumoniae was $71.4 \%$ (5 of 7), lower than the $77.8 \%$ (49 of 63) found among PCR-negative patients, but this difference was not significant $(p=0.66)$.

\section{DISCUSSION}

Respiratory infections with M. pneumoniae are common in most areas of the world, which cause atypical pneumonia and other respiratory tract diseases with outbreaks occurring every 4-7 year intervals. The annual rate of infection is estimated between $1.3 \%$ in endemic periods and $50 \%$ in epidemic periods. About $15-20 \%$ of community-acquired pneumonia was associated with $M$. pneumoniae, with an incidence of 2 cases per 1,000 population annually. ${ }^{25,26}$

The role of M. pneumoniae in respiratory infections was investigated in different regions, but its prevalence varies greatly from study to study. Although geographical regions can alter the prevalence of $M$. pneumoniae in respiratory infections, type of study, age of studied patients and the methods used for detecting M. pneumoniae can be responsible for the discrepant results of previous studies. Our study revealed that $10 \%$ of patients with LRTIs could be attributed to this atypical bacteria, which is similar to that one reported in other parts of Asia, ${ }^{27,28}$ in spite of the fact that our study was conducted in a different location and at a different time. Ngeow et al. carried on a large surveillance study in Asia, which showed the prevalence of $M$. pneumoniae in about $12 \%$ among children with community-acquired pneumonia. ${ }^{27}$ In an earlier study from South-eastern Asia, Ouchi et al. studied 1,104 Japanese children with acute LRTIs and found M. pneumoniae in $13 \%$ of the patients, ${ }^{28}$ also in agreement with our study.

Low frequency of $M$. pneumoniae was reported in only few studies, such as the study by Elkholy et al. involving 111 Egyptian children with positivity rate of only $4.5 \%{ }^{29}$ and the study by Meijer et al. on 557 Dutch patients with positivity rate of only $1.3 \%{ }^{30}$ However, there are several studies reporting higher frequency of $M$. pneumoniae. In the study by Principi et al. on 613 Italian children from 21 different centers, who were hospitalized because of community acquired LRTIs, this pathogen was found in $34 \%$ of the patients. ${ }^{31}$ In the study by Liu et al. on 256 Taiwanese patients with clinical suspicion of atypical pneumonia, M. pneumoniae was positive in $32 \%$ of the cases. ${ }^{24}$ In another study by Maltezou et al. on 65 Greek children, M. pneumoniae infection was confirmed in $27.5 \%$ of them. ${ }^{32}$ As expected, the frequency of M. pneumoniae pneumonia should be much higher at the time of outbreaks. The study by Kim et al. in Seoul, Korea, on 234 hospitalized children with community-acquired LRTIs during two outbreaks revealed about $65 \%$ of patients with M. pneumoniae pneumonia. ${ }^{33}$ 
However, it should be emphasized that some of these studies reflect the prevalence of M. pneumoniae in hospitalized patients, while we also included outpatients, albeit with similar frequency with hospitalized cases. Although the study by Sidal $e$ t al. in Istanbul, Turkey on 284 patients, seen at an outpatient clinic with respiratory symptoms, showed positive antibody for $M$. pneumoniae in about $30 \%$ of cases. ${ }^{34}$ Another study by Butun et al. in Izmir, Turkey on 100 children, receiving care at the outpatient department with respiratory symptoms, revealed positive antibody for $M$. pneumoniae in only $8 \%$ of cases. ${ }^{35}$

In our study, the $M$. pneumoniae in autumn season was found higher compared to other seasons, which contrasts with the Turkish study indicating significantly higher frequency of this pathogen in winter season. ${ }^{34}$ Meanwhile in the study by Foy et al. in Seattle during an 11-year period, no significant seasonal variation was documented. ${ }^{36}$

Atypical pneumonia could be distinguished from other types of pneumonias by its mild clinical course and its ability to cause different symptoms in different people. Cough and throat pain are common symptoms, whilst fever is usually a symptom of hospitalized patients diagnosed with pneumonia. However, we did not find any specific clinical characteristic that could accurately identify patients with M. pneumoniae, similarly to other studies. ${ }^{35,37}$ In fact, none of the signs and symptoms was unique to $M$. pneumoniae infections enabling to predict atypical pathogens based on clinical characteristics. Such findings can emphasize the point that clinical and laboratory features alone cannot predict the etiology of community-acquired pneumonia in children and therefore are not useful in therapeutic decision-making. ${ }^{38,39}$ It shows the importance of effective laboratory diagnosis tools to detect such pathogens. As clinical symptoms are nonspecific, persistent cough lasting more than a week, refractory to conventional antibiotic therapy should prompt clinicians to suspect the diagnosis of LRTIs by atypical microorganisms. ${ }^{40}$ However, initiation of effective antibiotic therapy is usually delayed. Such delay may lead to some complications, while the patients are not appropriately treated. ${ }^{31}$

Therefore, detection of microorganisms and appropriate treatments are the key points in the management of patients with LRTIs. Although M. pneumoniae can be isolated from cultures, this is a difficult and time-consuming method which limits its clinical usefulness. Therefore, the PCR method for detecting $M$. pneumoniae in throat swabs could be considered as the most practical method for rapid diagnosis, particularly in younger children and in early stage of disease..$^{20,24}$

M. pneumoniae is an atypical bacterium that could cause LRTIs worldwide, which requires adding a macrolide in the antibiotic regimen. ${ }^{40}$ Prompt diagnosis by appropriate technique and starting appropriate antibiotic therapy could prevent the use of unnecessary antibiotics and further complications.

\section{REFERENCES}

1. Mulholland K. Magnitude of the problem of childhood pneumonia. Lancet 1999; 354:590-2.

2. Vuori E, Peltola H, Kallio MJT et al. Etiology of pneumonia and other common childhood infections requiring hospitalization and parenteral antimicrobial therapy. Clin Infect Dis $1998 ; 27: 566-72$

3. Klig JE, Shah NB. Office pediatrics: current issues in lower respiratory infections in children. Curr Opin Pediatr 2005; 17:111-8.

4. Thomson RB. Laboratory diagnosis of respiratory infections. Curr Opin Infect Dis 1999; 12:115-9.

5. Menéndez R, Córdoba J, de la Cuadra $\mathrm{P}$ et al. Value of the polymerase chain reaction assay in noninvasive respiratory samples for diagnosis of community-acquired pneumonia. Am J Respir Crit Care Med 1999; 159:1868-73.

6. George H, Mccracken JR. Diagnosis and management of pneumonia in children. Pediatr Infect Dis J 2000; 19:924-8.

7. Mandell LA, Wunderink RG, Anzueto A et al. Infectious Diseases Society of America/American Thoracic Society consensus guidelines on the management of communityacquired pneumonia in adults. Clin Infect Dis 2007; 44:S2772.

8. Stolza D, Christ-Craind M, Gencaya MM et al. Diagnostic value of signs, symptoms and laboratory values in lower respiratory tract infection. Swiss Med Wkly 2006; 136:434-40.

9. Tan JS. Role of atypical pneumonia pathogens in respiratory tract infections. Can Respir J 1999; (Suppl 6):15-9.

10. Wadowsky RM, Castilla EA, Laus S et al. Evaluation of Chlamydia pneumoniae and Mycoplasma pneumoniae as etiologic agents of persistent cough in adolescents and adults. J Clin Microbiol 2002; 40:637-40.

11. File TM, Tan JS, Plouffe JF. The role of atypical pathogens: $M y$ coplasma pneumoniae, Chlamydia pneumoniae, and Legionella pneumophila in respiratory infection. Infect Dis Clin North Am 1998; 12:569-92.

12. Block S, Hedrick J, Hammerschlag MR et al. Mycoplasma pneumoniae and Chlamydia pneumoniae in pediatric community-acquired pneumonia: comparative efficacy and safety of clarithromycin vs. erythromycin ethylsuccinate. Pediatr Infect Dis J 1995; 14:471-7.

13. Taylor-Robinson D, Bebear C. Antibiotic susceptibilities of mycoplasmas and treatment of mycoplasmal infections. J Antimicrob Chemother 1997; 40:622-30.

14. Principi N, Esposito S. Comparative tolerability of erythromycin and newer macrolide antibacterials in paediatric patients. Drug Safety 1999; 20:25-41.

15. Harris R, Marmion BP, Varkanis G et al. Laboratory diagnosis of Mycoplasma pneumoniae infection. 2. Comparison of methods for the direct detection of specific antigen or nucleic acid sequences in respiratory exudates. Epidemiol Infect 1988; 101:685-94.

16. Lind K, Bentzon MW. Ten and a half years seroepidemiology of Mycoplasma pneumoniae infection in Denmark. Epidemiol Infect 1991; 107:189-99.

17. Petitjean J, Vabret A, Gouarin S, Freymuth F. Evaluation of four commercial immunoglobulin G (IgG)- and IgM-specific enzyme immunoassays for diagnosis of Mycoplasma pneumoniae infections. J Clin Microbiol 2002; 40:165-71.

18. Uldum SA, Søndergård-Andersen J, Skov Jensen J, Lind K. Evaluation of a commercial enzyme immunoassay for detection of Mycoplasma pneumoniae specific immunoglobulin G antibodies. Eur J Clin Microbiol Infect Dis 1990; 9:221-3. 
19. Echevarría JM, León P, Balfagón P et al. Diagnosis of Mycoplasma pneumoniae infection by microparticle agglutination and antibody-capture enzymeimmunoassay. Eur J Clin Microbiol Infect Dis 1990; 9:217-20.

20. Bernet C, Garret M, de Barbeyrac B et al. Detection of Mycoplasma pneumoniae by using the polymerase chain reaction. J Clin Microbiol 1989; 27:2492-6.

21. Ieven M, Ursi D, Van Bever H et al. Detection of Mycoplasma pneumoniae by two polymerase chain reactions and role of $M$. pneumoniae in acute respiratory tract infections in pediatric patients. J Infect Dis 1996; 173:1445-52.

22. Abele-Horn M, Busch U, Nitschko $\mathrm{H}$ et al. Molecular approaches to diagnosis of pulmonary diseases due to Mycoplasma pneumoniae. J Clin Microbiol 1998; 36:548-51.

23. Skakni L, Sardet A, Just J et al. Detection of Mycoplasma pneumoniae in clinical samples from pediatric patients by polymerase chain reaction. J Clin Microbiol 1992; 30:2638-43.

24. Liu FC, Chen PY, Huang F et al. Rapid diagnosis of Mycoplasma pneumoniae infection in children by polymerase chain reaction. J Microbiol Immunol Infect 2007; 40:507-12.

25. Foy HM, Cooney MK, McMahan R, Grayston JT. Viral and mycoplasmal pneumonia in a prepaid medical care group during an eight-year period. Am J Epidemiol 1973; 97:93-102.

26. Foy HM. Infections caused by Mycoplasma pneumoniae and possible carrier state in different populations of patients. Clin Infect Dis 1993; 17:S37-46.

27. Ngeow YF, Suwanjutha S, Chantarojanasriri T et al. An Asian study on the prevalence of atypical respiratory pathogens in community-acquired pneumonia. Int J Infect Dis 2005; 9:144-53.

28. Ouchi K, Komura H, Fujii M et al. Chlamydia pneumonia infection and Mycoplasma pneumoniae infection in pediatric patients. Kansenshogaku Zasshi 1999; 73: 1177-82.

29. Elkholy A, Elkaraksy H, Fattouh A et al. Acute lower respiratory tract infection due to Chlamydia and Mycoplasma spp. in Egyptian children under 5 years of age. J Trop Pediatr 2009; 55:195-7.

30. Meijer A, Dagnelie CF, De Jong JC et al. Low prevalence of Chlamydia pneumoniae and Mycoplasma pneumoniae among patients with symptoms of respiratory tract infections in Dutch general practices. Eur J Epidemiol 2000; 16:1099-106.
31. Principi N, Esposito S, Blasi F, Allegra L, Mowgli Study Group. Role of Mycoplasma pneumonia and Chlamydia pneumonia in children with community-acquired lower respiratory tract infections. Clin Infect Dis 2001; 32:1281-9.

32. Maltezou HC, La-Scola B, Astra H et al. Mycoplasma pneumoniae and Legionella pneumophila in community-acquired lower respiratory tract infections among hospitalized children: diagnosis by real time PCR. Scand J Infect Dis 2004; 36:639-42.

33. Kim NH, Lee JA, Eun BW et al. Comparison of polymerase chain reaction and the indirect particle agglutination antibody test for the diagnosis of Mycoplasma pneumoniae pneumonia in children during two outbreaks. Pediatr Infect Dis J 2007; 26:897-903.

34. Sidal M, Kilic A, Unuvar E et al. Frequency of Chlamydia pneumoniae and Mycoplasma pneumoniae infections in children. J Trop Pediatr 2007; 53:225-31.

35. Bütün Y, Köse S, Babayiğit A et al. Chlamydia and Mycoplasma serology in respiratory tract infections of children. Tuberk Toraks 2006; 54:254-8.

36. Foy HM, Kenny GE, Cooney MK et al. Longterm epidemiology of infections with Mycoplasma pneumoniae. J Infect Dis 1979; 139:681-7.

37. Esposito S, Blasi F, Bellini F et al. Mycoplasma pneumoniae and Chlamydia pneumoniae infections in children with pneumonia. Eur Respir J 2001; 17:241-5.

38. Wubbel L, Muniz L, Ahmed A et al. Etiology and treatment of community-acquired pneumonia in ambulatory children. Pediatr Infect Dis J 1999; 18:98-104.

39. Korppi M, Heiskanen-Kosma T, Jaloner E et al. Aetiology of community-acquired pneumonia in children treated in hospital. Eur J Pediatr 1993; 152:24-30.

40. Esposito S. Importance of acute Mycoplasma pneumoniae and Clamydia pneumoniae infections in children with wheezing. Eur Respir J 2000; 16:1142-6. 